LA WRENCE LWWEAMCAE NATIONAL LABDPATOAY

Ab initio shell model with a chiral-symmetry-based three-nucleon force for the p-shell nuclei

P. Navratil, A.C. Hayes, J.P. Vary, and W.E. Ormand

September 25, 2003 
This document was prepared as an account of work sponsored by an agency of the United States Government. Neither the United States Government nor the University of California nor any of their employees, makes any warranty, express or implied, or assumes any legal liability or responsibility for the accuracy, completeness, or usefulness of any information, apparatus, product, or process disclosed, or represents that its use would not infringe privately owned rights. Reference herein to any specific commercial product, process, or service by trade name, trademark, manufacturer, or otherwise, does not necessarily constitute or imply its endorsement, recommendation, or favoring by the United States Government or the University of California. The views and opinions of authors expressed herein do not necessarily state or reflect those of the United States Government or the University of California, and shall not be used for advertising or product endorsement purposes.

This work was performed under the auspices of the U.S. Department of Energy by the University of California, Lawrence Livermore National Laboratory under Contract W7405-Eng-48. 


\title{
Ab initio shell model with a chiral-symmetry-based three-nucleon force for the $p$-shell nuclei
}

\author{
P. Navratil ${ }^{\text {* }}$, A. C. Hayes ${ }^{\mathrm{b}}$, J. P. Vary ${ }^{\mathrm{c}}$ and W. E. Ormand ${ }^{\mathrm{a}}$ \\ ${ }^{a}$ Lawrence Livermore National Laboratory, \\ L-414, P. O. Box 808, Livermore, California 94551, USA \\ b'Theoretical Division, Los Alamos National Laboratory, \\ Los Alamos, New Mexico 87545, USA
}

${ }^{\mathrm{c}}$ Department of Physics and Astronomy, Iowa State University, Ames, Iowa 50011, USA

The $a b$ initio no-core shell model (NCSM) is extended to include a realistic three-body interaction in calculations for $p$-shell nuclei. We present results of first applications using the Argonne V8' nucleon-nucleon (NN) potential and the Tucson-Melbourne TM'(99) three-nucleon interaction (TNI). In addition to increase of binding energy, we observe a trend toward level-ordering and level-spacing improvement in comparison to experiment. With the TNI we obtain a correct ground-state spin for ${ }^{10} \mathrm{~B}$ contrary to calculations with NN potentials only. We also investigate neutrino- ${ }^{12} \mathrm{C}$ exclusive cross sections and muon capture on ${ }^{12} \mathrm{C}$. We show that realistic nucleon-nucleon interactions underpredict the experimental cross sections by a factor of two or more. By including the TNI a much better agreement with experiment is achieved along with an encouraging trend.

\section{Ab initio No-Core Shell Model with a Genuine Three-Body Interaction}

It is well established that the modern accurate NN potentials must be augmented by realistic three-body interactions in order to reproduce experimental binding energies, scattering observables and nuclear structure of $A>2$ nuclei.

In this paper, we briefly present extensions and modifications of the NCSM $[1,2]$ needed when a genuine TNI is included as well as results of first applications. The starting Hamiltonian is $H_{A}=\frac{1}{A} \sum_{i<j} \frac{\left(\vec{p}_{i}-\vec{p}_{j}\right)^{2}}{2 m}+\sum_{i<j}^{A} V_{\mathrm{NN}, i j}+\sum_{i<j<k}^{A} V_{\mathrm{NNN}, i j k}$. We employ a large but finite harmonic-oscillator (HO) basis. Due to properties of the realistic nuclear interaction we have to derive an effective interaction appropriate for the selected finite basis space. To facilitate this, we modify the Hamiltonian by adding to it (and subtracting from it in the end) the center-of-mass (CM) HO Hamiltonian $H_{\mathrm{CM}}=T_{\mathrm{CM}}+U_{\mathrm{CM}}$, where $U_{\mathrm{CM}}=\frac{1}{2} A m \Omega^{2} \vec{R}^{2}, \vec{R}=\frac{1}{A} \sum_{i=1}^{A} \vec{r}_{i}$. The modified Hamiltonian is then $H_{A}^{\Omega}=H_{A}+H_{\mathrm{CM}}=$ $\sum_{i=1}^{A} h_{i}+\sum_{i<j}^{A} V_{i j}^{\Omega, A}+\sum_{i<j<k}^{A} V_{\mathrm{NNN}, i j k}$, with $h_{i}=\frac{\vec{p}_{i}^{2}}{2 m}+\frac{1}{2} m \Omega^{2} \vec{r}_{i}^{2}$ and $V_{i j}^{\Omega, A}=V_{\mathrm{NN}, i j}-\frac{m \Omega^{2}}{2 A}\left(\vec{r}_{i}-\right.$

\footnotetext{
${ }^{*}$ This work was performed in part under the auspices of the U. S. Department of Energy by the University of California, Lawrence Livermore National Laboratory under contract No. W-7405-Eng-48.

${ }^{\dagger}$ This work was partly supported by U.S. DOE FG-02-87ER40371.
} 
$\left.\vec{r}_{j}\right)^{2}$. Next we divide the $A$-nucleon infinite $\mathrm{HO}$ basis space into the finite active space $P$ comprising all states up to $N_{\max } \mathrm{HO}$ excitations above the unperturbed ground state and the excluded spaces $Q$. The basic idea of the NCSM approach is to apply a unitary transformation on the modified Hamiltonian, $e^{-S} H_{A}^{\Omega} e^{S}$ such that $Q e^{-S} H_{A}^{\Omega} e^{S} P=0$. We apply this approach with a cluster approximation. When a genuine TNI is considered, the simplest cluster approximation produces a three-body effective interaction. The NCSM calculation is then performed in four steps:

(i) We solve a three-nucleon system for all possible three-nucleon channels with the Hamiltonian $H_{A}^{\Omega}$,i.e., using $h_{1}+h_{2}+h_{3}+V_{12}^{\Omega, A}+V_{13}^{\Omega, A}+V_{23}^{\Omega, A}+V_{\mathrm{NNN}, 123}$.

(ii) We construct the unitary transformation from the three-nucleon solutions using the Lee-Suzuki procedure [3] and calculate the three-body effective interaction.

(iii) As the three-body effective interaction is derived in the Jacobi-coordinate HO basis but the $p$-shell calculations are performed in a Cartesian-coordinate single-particle basis, we need to perform a suitable transformation of the interaction.

(iv) We solve the Schrödinger equation for the $A$-nucleon system using a Hamiltonian that includes the derived three-body effective interaction.

\section{Application to ${ }^{10} \mathrm{~B}$}

Our ${ }^{10} \mathrm{~B}$ excitation energy results are shown in Fig. 1. We note that our calculations with realistic two-nucleon interactions predict an incorrect ground-state spin in this nucleus [2], i.e., $1_{1}^{+} 0$ instead of the experimental $3_{1}^{+} 0$. The same prediction is obtained by the GFMC method [5]. Our results obtained using the $\mathrm{TM}^{\prime}(99)$ TNI indeed show that the three-nucleon interaction has a positive impact on the excitation spectrum and corrects the level ordering by bringing the $3_{1}^{+} 0$ state below the $1_{1}^{+} 0$ state in agreement with experiment. In the GFMC calculations of Ref. [5], the correct ${ }^{10} \mathrm{~B}$ ground state was obtained using the Illinois TNI but the $1_{1}^{+} 0$ ground state remained when the Urbana IX TNI was employed. Combined with our observed sensitivity for higher excited states this shows that nuclear structure will play an important role in determining the form and the parametrization of the three-nucleon interaction that is still not well known.

\section{Neutrino scattering on ${ }^{12} \mathbf{C}$}

The transition from the ${ }^{12} \mathrm{C}_{\text {g.s. }}$ to the $\mathrm{T}=11^{+}$states in mass 12 is very sensitive to the strength of the spin orbit interaction. Using our NCSM wave functions, we calculated exclusive ${ }^{12} \mathrm{C}_{\mathrm{gs}} \rightarrow{ }^{12} \mathrm{~N}_{\mathrm{gs}}\left(\nu_{e}, e^{-}\right)$and $\left(\nu_{\mu}, \mu^{-}\right)$neutrino cross sections as well as muon capture on ${ }^{12} \mathrm{C}$ (see Table 1). These processes are characterized by vastly different energies and momentum transfers. The neutrino spectrum for electron neutrinos from decay-at-rest (DAR) of the pion peaks around $30 \mathrm{MeV}$ while the $\left(\nu_{\mu}, \mu^{-}\right)$neutrino cross section to ${ }^{12} \mathrm{~N}_{\text {g.s. }}$. corresponds to muon neutrinos from decay-in-flight (DIF) of the pion. This spectrum involves neutrinos with an average energy of about $150 \mathrm{MeV}$. Muon capture involves an average momentum transfer of $q \sim 100 \mathrm{MeV} / \mathrm{c}$. The CD-Bonn interaction (without TNI) results indicate an approach to convergence by $6 \hbar \Omega$ but experiment is underpredicted by about a factor of 2 or more. When the TNI is included with the AV8' interaction the predicted DAR cross section and muon capture rate is only about $30 \%$ lower than experiment while the DIF cross section is in fact in agreement with experiment. 


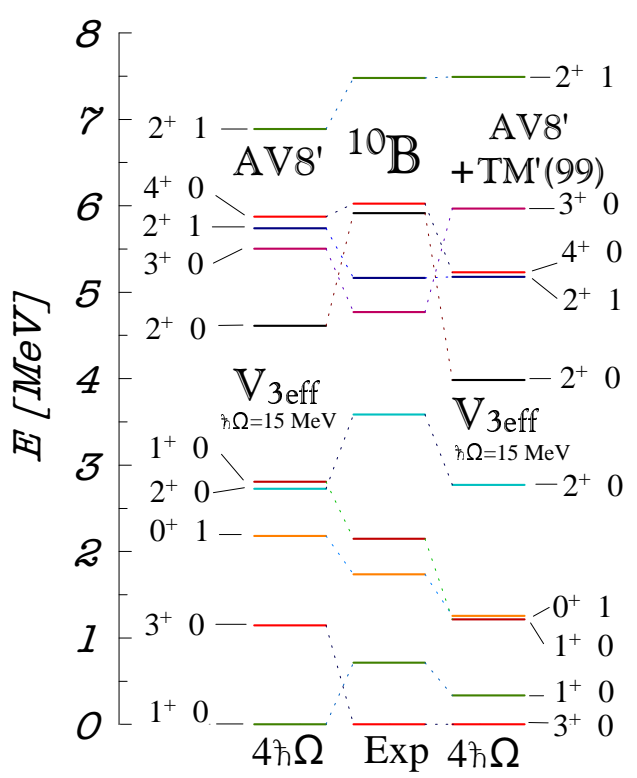

Figure 1. Levels of ${ }^{10} \mathrm{~B}$ obtained using $\mathrm{AV} 8^{\prime}$ and $\mathrm{AV}^{\prime}+\mathrm{TM}^{\prime}(99)$ interactions.

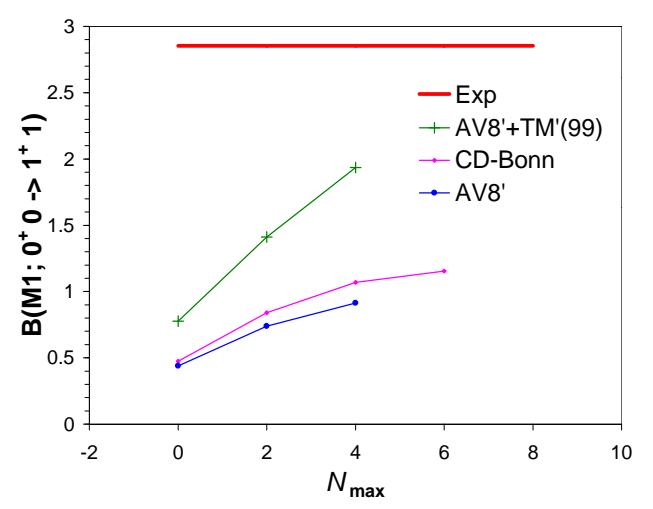

Figure 2. $\mathrm{B}(\mathrm{M} 1)$ values, in $\mu_{N}^{2}$, of the $0^{+} 0 \rightarrow 1^{+} 1$ transition in ${ }^{12} \mathrm{C}$.

Table 1

Predicted weak interaction rates for the ${ }^{12} \mathrm{C} \rightarrow \mathrm{T}=11^{+}$transitions. The units are $10^{-42} \mathrm{~cm}^{2}\left(\left(\nu_{e}, e^{-}\right) \mathrm{DAR}\right), 10^{-40} \mathrm{~cm}^{2}\left(\left(\nu_{\mu}, \mu^{-}\right) \mathrm{DIF}\right)$ and $10^{3} \mathrm{sec}^{-1}$ ( $\mu$-capture).

\begin{tabular}{c|ccccc}
\hline Interaction & CD-Bonn & CD-Bonn & CD-Bonn & $\mathrm{AV8}^{\prime}+\mathrm{TM}^{\prime}(99)$ & experiment \\
Basis space & $2 \hbar \Omega$ & $4 \hbar \Omega$ & $6 \hbar \Omega$ & $4 \hbar \Omega$ & \\
\hline$\left(\nu_{e}, e^{-}\right)$ & 2.27 & 3.2 & 3.69 & 6.8 & $8.9 \pm 0.3 \pm 0.9[6]$ \\
$\left(\nu_{\mu}, \mu^{-}\right)$ & 0.168 & 0.275 & 0.312 & 0.537 & $0.56 \pm 0.08 \pm 0.1[7]$ \\
$\mu$-capture & 1.46 & 2.07 & 2.38 & 4.43 & $6.0 \pm 0.4[8]$ \\
\hline
\end{tabular}

The conclusion drawn from the neutrino cross section results is further supported by our $\mathrm{B}\left(\mathrm{M} 1 ; 0^{+} 0 \rightarrow 1^{+} 1\right)$ results presented in Fig. 2. The calculations with 2-body forces show saturation and underpredict the experiment by almost a factor of three. By including the TNI, the $\mathrm{B}(\mathrm{M} 1)$ value increases dramatically.

\section{REFERENCES}

1. P. Navrátil, J. P. Vary and B. R. Barrett, Phys. Rev. C 62 (2000) 054311.

2. P. Navrátil and W. E. Ormand, Phys. Rev. Lett. 88 (2002) 152502.

3. K. Suzuki and S. Y. Lee, Prog. Theor. Phys. 64 (1980) 2091.

4. S. A. Coon and H. K. Han, Few-Body Systems 30 (2001) 131.

5. S. C. Pieper, K. Varga and R. B. Wiringa, Phys. Rev. C 66 (2002) 044310.

6. LSND collaboration, L. B. Auerbach et al., Phys. Rev. C 64 (2001) 065501.

7. LSND collaboration, L. B. Auerbach et al., Phys. Rev. C 66 (2002) 015501.

8. M. Giffon et al., Phys. Rev. C 24 (1981) 241. 